\title{
BMJ Open Swallowing behaviours and feeding environment in relation to communication development from early infancy to 6 years of age: a scoping review protocol
}

\author{
Heather Flowers, ${ }^{01,2}$ Daniel Bérubé, ${ }^{1}$ Mona Ebrahimipour, ${ }^{1,3}$ \\ Marie-France Perrier, ${ }^{1,4}$ Sarah Moloci, ${ }^{5}$ Stacey Skoretz ${ }^{6,7}$
}

To cite: Flowers H, Bérubé D,

Ebrahimipour M, et al.

Swallowing behaviours and feeding environment in relation to communication development from early infancy to 6 years of age: a scoping review protocol. BMJ Open 2019;9:e028850. doi:10.1136/ bmjopen-2018-028850

- Prepublication history and additional material for this paper are available online. To view these files, please visit the journal online (http://dx.doi org/10.1136bmjopen-2018028850).

Received 27 December 2018 Revised 25 June 2019 Accepted 18 July 2019
Check for updates

(C) Author(s) (or their employer(s)) 2019. Re-use permitted under CC BY-NC. No commercial re-use. See rights and permissions. Published by BMJ.

For numbered affiliations see end of article.

Correspondence to Dr Heather Flowers; heather.flowers@uottawa.ca

\section{ABSTRACT}

Introduction Understanding the influences of early swallowing function and feeding environment on the development of communication will enhance prevention and intervention initiatives for young children. This scoping review will help elucidate key elements affecting the developmental trajectory of communicative systems, typically robust and well-developed by formal school entry. We aim to (1) map the current state of the literature in a growing field of interest that has the potential to advance knowledge translation, (2) identify existing gaps and (3) provide research direction for future investigations surrounding feeding-swallowing functions and environment that support or forestall communication development in young children.

Methods and analysis We are proposing a scoping review to identify the breadth and depth of the existing literature regarding swallowing-feeding functions and environment relative to the onset and progression of communicative behaviours from infancy to $6(<6 ; 0)$ years of age. Our protocol delineates rigorous methods according to Arskey and 0'Malley's framework and includes elaborations by Levac and colleagues. We will search the literature based on 10 databases, 17 peerreviewed journals, 4 conference proceedings and 6 grey literature sources. Two authors will independently screen abstracts and review full articles, remaining blind to each other's results. A third author will contribute to resolving any discrepant results from both the abstract and article review. Subsequently, we will extract data and chart information from accepted articles using a pre-established data collection form. We will stratify results according to healthy versus impaired swallowing-feeding functions and communication development.

Ethics and dissemination Our scoping review does not require ethical approval. We will disseminate our final study results through international and national conference presentations, publication in a peer-reviewed journal and knowledge translation activities with stakeholders.

\section{BACKGROUND}

There has been growing interest in the effects of swallowing and feeding functions on the
Strengths and limitations of this study

- A key strength includes a first and comprehensive literature mapping for research on the influence of early swallowing-feeding behaviours and environment on communication development.

- A second strength includes a rigorous search design involving multiple sources, such as databases, journals, conference proceedings and the grey literature.

- An inherent limitation of our scoping review is that we will not conduct quality appraisal for individual articles given anticipated breadth of results and research designs.

- A second limitation is that we did not include nutritional factors alongside feeding as potential mediators of optimal development of communicative behaviours. The breadth of the review protocol is extensive and cannot feasibly accommodate infant or maternal nutritional inquiry.

development of communicative systems in young children. Research is necessary to understand the complexity of influences that may foster optimal development and conversely forestall delays, especially in the wake of increasingly prevalent communication disorders. Whether or not children incur communication disorders via elusive causes or predisposing conditions, a high proportion of newborn babies will develop a communication disorder. They often remain vulnerable from birth through to school entry (generally before the age of 6 years), especially if diagnosis is delayed or early interventions are lacking. ${ }^{1}$ Various prenatal, perinatal or postnatal congenital abnormalities or conditions, including syndromes, cerebral palsy and premature birth, may predispose children to impaired communication development. Currently, congenital syndromes affect about 


\begin{tabular}{|c|c|c|}
\hline Item & Term set & Area \\
\hline 1 & exp Deglutition/ & Swallowing \\
\hline 2 & exp Gastroesophageal reflux/ & Swallowing \\
\hline 3 & Deglutition disorders/ & Swallowing \\
\hline 4 & 'Feeding and eating disorders of childhood'/ & Swallowing \\
\hline 5 & Breast feeding/ & Swallowing \\
\hline 6 & Bottle feeding/ & Swallowing \\
\hline 7 & Feeding methods/ & Swallowing \\
\hline 8 & Enteral nutrition/ & Swallowing \\
\hline 9 & Parenteral nutrition/ & Swallowing \\
\hline 10 & Kangaroo-mother care method/ & Swallowing \\
\hline 11 & Pacifiers/ & Swallowing \\
\hline 12 & sucking behaviors/ & Swallowing \\
\hline 13 & ((enteral or tube or gastric) adj (feed\$)).ti,ab,kw. & Swallowing \\
\hline 14 & $\begin{array}{l}\text { ((deglut } \$ \text { or feed } \$ \text { or swallow } \$ \text { or feed } \$ \text {-swallow } \$ \text { ) adj3 (difficult } \$ \text { or disorder } \$ \text { or abnormal } \$ \text { or delay } \$ \text { or } \\
\text { dysfunction } \$ \text { or impair } \$ \text { or problem } \$ \text { or disabil } \$ \text { or disabl } \$ \text { or deficit } \$) \text { ) .ti,ab, } \mathrm{kw} \text {. }\end{array}$ & Swallowing \\
\hline 15 & $\begin{array}{l}\text { ((deglut } \$ \text { or feed } \$ \text { or swallow } \$ \text { or feed } \$ \text {-swallow } \$ \text { ) adj3 (behavior or develop } \$ \text { or neurodevelop } \$ \text { or } \\
\text { matur } \$ \text { or function } \$) \text { ).ti,ab, kw. }\end{array}$ & Swallowing \\
\hline 16 & (dysphag\$).ti,ab,kw. & Swallowing \\
\hline 17 & ((bottle fe\$) or (bottlefe\$) or (breast fe\$) or (breastfe\$) or (feeding mode) or (feeding methods)).ti,ab,kw. & Swallowing \\
\hline 18 & ((gastroesophageal or gastro-esophageal or infantile or gastric) adj3 (reflux or regurgitat\$)).ti,ab,kw. & Swallowing \\
\hline 19 & exp Speech/ & Comm \\
\hline 20 & exp Speech disorders/ & Comm \\
\hline 21 & exp Language development disorders/ & Comm \\
\hline 22 & exp Child language/ & Comm \\
\hline 23 & Speech sound disorder/ & Comm \\
\hline 24 & Verbal behavior/ & Comm \\
\hline 25 & Manual communication/ & Comm \\
\hline 26 & Communication disorders/ & Comm \\
\hline 27 & Social communication disorder/ & Comm \\
\hline 28 & Autism spectrum disorder/ & Comm \\
\hline 29 & Language disorders/ & Comm \\
\hline 30 & Language development/ & Comm \\
\hline 31 & $\begin{array}{l}\text { ((speech or oromotor or "oral motor" or verbal\$ or oro-motor or orofacial or oro-facial or "oral facial") adj3 } \\
\text { (aprax\$ or dysprax\$ or prax\$)).ti,ab,kw. }\end{array}$ & Comm \\
\hline 32 & $\begin{array}{l}\text { ((speech or speak } \$ \text { or oral or language or verbal } \$ \text { or communication or voice or vocal } \$ \text { or babbl} \$ \text { or coo } \$ \\
\text { or "oral motor" or oromotor or oro-motor or orofacial or oro-facial or "oral facial" or oralfacial or articulat } \$ \\
\text { or phonetic } \$ \text { or phonologic } \$ \text { or phonemic } \$ \text { or pre-linguistic or prelinguistic) adj3 (difficult } \$ \text { or disorder } \$ \text { or } \\
\text { abnormal } \$ \text { or delay } \$ \text { or dysfunction } \$ \text { or impair } \$ \text { or problem } \$ \text { or disabil } \$ \text { or disabl } \$ \text { or deficit } \$) \text { ).ti,ab,kw. }\end{array}$ & Comm \\
\hline 33 & $\begin{array}{l}\text { ((speech or speak } \$ \text { or oral or language or verbal\$ or communication or voice or vocal\$ or babbl\$ or coo } \$ \\
\text { or "oral motor" or oromotor or oro-motor or orofacial or oro-facial or "oral facial" or oralfacial or articulat\$ } \\
\text { or phonetic } \$ \text { or phonologic } \$ \text { or phonemic } \$ \text { or pre-linguistic or prelinguistic) adj3 (behavior or develop } \$ \text { or } \\
\text { neurodevelop } \$ \text { or matur } \$ \text { or acqui } \$)) . t i, a b, k w .\end{array}$ & Comm \\
\hline 34 & exp child/ & Population \\
\hline 35 & exp infant/ & Population \\
\hline 36 & adolescent/ & Population \\
\hline 37 & 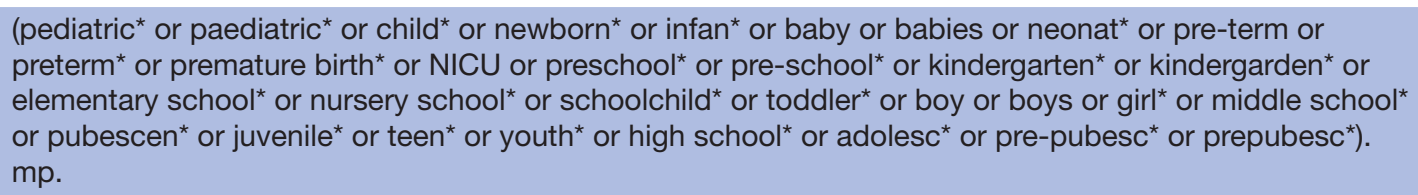 & Population \\
\hline
\end{tabular}


$4 \%$ of Canadian babies. ${ }^{2}$ In particular, the birth prevalence of Down syndrome is $0.16 \%{ }^{3}$ and orofacial clefts are evidenced in $0.13 \%$ of live births. ${ }^{4}$ Even more prevalent, cerebral palsy ranges from $0.31 \%$ to $0.36 \%$ of 8 -year-old children in the USA. ${ }^{5}$ Far exceeding the prevalence of other conditions, prematurity is currently estimated at $8 \%$ of all births, ${ }^{6}$ and ensuing difficulties may persist throughout childhood.

In fact, $8 \%$ of American children aged 3-17 years has a speech, language, voice or swallowing disorder. ${ }^{7}$ More specifically, a recent population study demonstrated a frequency of language disorders by entry to mainstream schooling in $10 \%$ of children. ${ }^{8}$ Close to one-third of the sample had a language disorder of unknown cause, ${ }^{8}$ which is often attributable to specific language impairment. ${ }^{9}$ Similarly, the prevalence of language impairment in Canadian kindergarteners is about $8 \% .{ }^{10}$ Concerning speech acquisition, $15 \%$ of 3-year-old and $4 \%$ of 6 -year-old children have a speech sound disorder. ${ }^{11}$ Speech and/or language impairments often co-occur, affecting over $10 \%$ of kindergarten children. ${ }^{10}$ They may be also present in children with social communication impairments, such as autism spectrum disorder, which affects one and a half percent of Canadian children currently. ${ }^{12}$

Despite good epidemiological information and a long history of research on speech and language development in the preschool years, ${ }^{13-17}$ a significant gap exists concerning knowledge of the earliest precursors to communication. ${ }^{13}$ There is, however, emerging evidence for synchrony and congruence between maternal vocalisations and fetal behaviours. ${ }^{18}$ In particular, the fetus is preferentially sensitive to particular types of maternal linguistic input and may even mirror associated gestural oral behaviours (such as mouth opening). ${ }^{19}$ Early after birth, synchronous mother-infant vocal behaviours continue to manifest. ${ }^{20}$ Vocalisations of the newborn infant include cries, vegetative sounds and comfort sounds such as cooing, as described in Stark and her colleague's ${ }^{21}$ seminal study, all occurring well before the onset of reduplicated babbling. ${ }^{13}$ Of note, a recent study underscored differences in maternal response to early speech-like vocalisations (protophones) compared with infant cries. ${ }^{20}$ That is, mothers more frequently engaged in verbal turn-taking when infants produced protophones compared with cries. ${ }^{20}$ Cooing is an early speech-like behaviour, occurring as early as 1 month of age, ${ }^{21}$ and it may well be a hallmark indicator to the healthy progression of higher order speech and communicative systems because of its association with positive affect and relational interactions. ${ }^{22}$

A body of evidence suggests that early positive attachment and interactive experiences between mothers and babies facilitate affective, cognitive and communication development. ${ }^{23}$ Over the long term, children demonstrate improved social-emotional ${ }^{24} 25$ and cognitive ${ }^{26}$ development when their mothers are emotionally available, ${ }^{24} 26$ provide skin-to-skin contact ${ }^{25}$ and/or provide verbal stimulation ${ }^{26}$ in early infancy. Likewise, intact early infant neuroregulation, particularly attention (a precursor to interactive engagement), predicts good social communication outcomes by school entry. ${ }^{27}$

Notwithstanding, the nature and potential range of early facilitators and associated trajectory of infant responses that converge for the healthy or optimal development of communication, particularly the onset of joint attention, ${ }^{28}$ remain elusive. Given the highly responsive and largely reflexive behaviours in the neonatal period, young infants are difficult to evaluate and monitor in a natural setting. Hence, there remains a paucity of literature on very early onset of vocalisations and progression towards the development of symbolic communication despite evidence for early maternal verbal contingencies following infant vocalisations ${ }^{20} 29$ and interactive cues such as eye-gaze patterns. ${ }^{29}$ An ideal naturalistic early context for understanding communication development includes physiologically driven periods when infants are alert, most notably prior to and during feeding sequences. For example, early vocalisations of contentment may be inextricably linked to the feeding context. ${ }^{30}$ Interestingly, infants remain by and large dependent for feeding, until just about the time when symbolic communication and joint attention emerge (8-12 months) ${ }^{31}$ Consequently, the feeding environment can promote optimal linguistic exposure and interactions, facilitating development of the auditory system and various basic motor functions of the vocal tract. ${ }^{32}$

Various theoretical premises exist regarding the development of communication: (1) one revolving around the tightly coordinated and integrative physiological development of the respiratory and vocal/articulatory systems, ${ }^{33} 34$ (2) another particularly centred on intrinsic innate developmental stages for speech and language acquisition ${ }^{35} 36$ and (3) a third pertaining to a connectionist framework for speech production and/or processing alongside learning demands, ${ }^{37}$ dependent on capacity for resource allocation. ${ }^{38}$ By extension, a connectionist model could intuitively include physiological integrity as necessary for higher-level resource allocation, whereby excessive demands or dysfunction would preclude optimal activation of complex behaviours and cognitive processing. Conversely, physiological integrity coupled with environmental facilitators would permit the necessary resource allocation for the optimal development of integrated networks for communicative systems, ensuring appropriate stage to stage developmental progression.

To illustrate, from a physiological standpoint, both respiration and swallowing are primordial life-sustaining functions, and they are inextricably linked and tightly coordinated behaviours. ${ }^{39-41}$ Consequently, integrity of swallowing depends on adequate respiratory functions and together they provide the physiological and neurological basis for developing voice and speech functions. ${ }^{33} 34$ Physiological integrity of swallowing coupled with a facilitative feeding environment ${ }^{32}$ may well optimise connexions and prime the development of neural networks for communication. Conversely, detriment to swallowing 
Table 2 Complete list of search sources including databases, journals, conference proceedings and grey literature

\begin{tabular}{|c|c|}
\hline Literature type & Sources \\
\hline Databases & $\begin{aligned} & \text { AMED } \\
& \text { CINHAL } \\
& \text { Education Source } \\
& \text { Embase } \\
& \text { ERIC } \\
& \text { Linguistics and Language Behaviour } \\
& \text { Abstracts } \\
& \text { Medline } \\
& \text { Psyclnfo } \\
& \text { Scopus } \\
& \text { Web of Science }\end{aligned}$ \\
\hline Journals & $\begin{array}{l}\text { American Journal of Speech-Language } \\
\text { Pathology } \\
\text { Canadian Journal of Speech-Language } \\
\text { Pathology } \\
\text { Child Development } \\
\text { Clinical Linguistics and Phonetics } \\
\text { Developmental Medicine and Child } \\
\text { Neurology } \\
\text { Dysphagia } \\
\text { Gastroenterology } \\
\text { Infancy } \\
\text { International Journal of Behavioral } \\
\text { Development } \\
\text { International Journal of Speech- } \\
\text { Language Pathology } \\
\text { Journal of Child Language } \\
\text { Journal of Communication Disorders } \\
\text { Journal of Pediatric Gastroenterology } \\
\text { and Nutrition } \\
\text { Journal of Pediatrics } \\
\text { Journal of Speech, Language, and } \\
\text { Hearing Research } \\
\text { Nature } \\
\text { The American Journal of } \\
\text { Gastroenterology }\end{array}$ \\
\hline $\begin{array}{l}\text { Conference } \\
\text { Proceedings }\end{array}$ & $\begin{array}{l}\text { ASHA Conference } \\
\text { Dysphagia Research Society } \\
\text { International Conference on Speech } \\
\text { and Language Development } \\
\text { World Pediatrics }\end{array}$ \\
\hline Grey Literature & $\begin{array}{l}\text { Networked Digital Library of Theses } \\
\text { and Dissertations } \\
\text { Open Access Theses and Dissertations } \\
\text { Open Grey } \\
\text { Proquest Dissertations and Theses } \\
\text { Global } \\
\text { Ontario Public Health Libraries } \\
\text { Association } \\
\text { - Grey Matters }\end{array}$ \\
\hline
\end{tabular}

functions can incur disease and grossly compromise physiological integrity of the respiratory and auditory systems. Examples include increased risk of secondary respiratory infections ${ }^{42}$ and otitis media. ${ }^{434}$ There is a need to collect additional knowledge about swallowing/feeding and speech/language relationships, including mediation by the auditory system ${ }^{45}$ to help us improve our current understanding of healthy versus disordered development of communication. Specifically, we want to improve the processes of early detection, evaluation and intervention for swallowing/feeding and associated communicative functions in an integrated manner.

\section{Rationale}

Identifying relationships between early physiological functions (such as feeding and swallowing) and communication (from speech-like behaviours such as cooing to phonological development for meaningful word production and language use in discourse) will help us improve our understanding of the development of communication. We need to consider these relationships starting in early infancy because the feeding context has great potential as a facilitative environment for social interactions. Nevertheless, problems with swallowing function and/ or suboptimal feeding environment may have a grossly prohibitive effect on optimal and healthy development of communicative systems. We still need a comprehensive understanding of precursors and determinants longitudinally from birth to the age of 6 years, when most children begin formal schooling.

\section{Objectives}

The primary objective of our scoping review is to provide an overview of the literature considering the association between swallowing physiology and feeding environment and the development of communicative functions, whether healthy or disordered. The specific objectives include (1) identifying the number and design of articles over time, (2) mapping sample characteristics and study themes in the existing literature and (3) identifying gaps in knowledge pertaining to the contribution of swallowing function and feeding environment to the development of communicative systems in infants and young children.

\section{METHODS AND ANALYSIS Operational definitions}

We have defined swallowing to include oropharyngeal and/or oesophageal stages ${ }^{46}$ involving anatomical, neurological (eg, coordinated respiratory/swallowing behaviours) and/or physiological functions. Paediatric feeding includes (1) interest in feeding ${ }^{47}$ and consequent environmental engagement, (2) behavioural manifestations of dietary preferences and/or (3) progression through developmentally appropriate food and/or liquid consistencies and type. ${ }^{48}$ As such, feeding does not include nutritional aspects of intake and/or diet, such as adequacy of macro or micronutrients. Hereafter, the term 'environment' will refer to contingent internal and external stimuli within the feeding process and related context. Consequently, elements could include feeding mode, accompanying auditory or visual stimuli, location 
Table 3 Proposed coding categories for abstract and full article review

\section{Step 1: exclude if abstract}

(1) Is clearly a review, commentary or opinion

(2) Clearly has only an adult sample ( $\geq 18$ years of age)

(3) Swallowing/feeding context outside operational definition (ie, relates exclusively to nutritional intake)

(4) Outcomes relating to communication or other aspects of child development (motor, cognitive) clearly absent
(5) $n / a$

(6) Clearly involves same data as another abstract Otherwise accept for full article retrieval

\section{Step 2: exclude if full article}

(1) Is clearly a review, commentary or opinion

(2) Has a sample exclusively outside desired age range ( $\geq 6$ years of age)

(3) Swallowing/feeding context outside operational definition (ie, relates exclusively to nutritional intake)

(4) Outcomes relating to communication clearly absent

(5) Outcomes relating to swallowing/feeding and communication development clearly absent

(6) Clearly involves same data as another article Otherwise accept for scoping review (eg, intensive care unit, home, an incubator) and social interactions (eg, verbal and tactile).

Communication refers to the exchange of information, ideas, needs and desires between at least two persons. ${ }^{31}$ It involves both linguistic and paralinguistic codes, ${ }^{31}$ whereby methods of transmission include speech, intonation, gestures and body language. ${ }^{31}$ Examples of earliest behavioural manifestations may include first words or manual signs, presenting in children as young as 8 months, ${ }^{31}$ rapidly increasing in complexity. ${ }^{49}$ For the purpose of this scoping review, the definition of communication encapsulates the entire developmental progression from earliest precursors in the newborn infant to the use of near adult-like constructs by school entry. ${ }^{31}$

Therefore, we need to appreciate factors involving swallowing physiology and feeding relative to the onset of prelinguistic vocalisations and progression towards a highly complex communicative system. Such an understanding will help identify early facilitators or prognostic indicators of delay in the development of communication, whether speech, language or social communication (or a combination therein). A recent shift in culture in healthcare settings has already manifested, whereby early infant behaviours (such as vocalisations) are understood to be cues that communicate a need and warrant a social interactive response. ${ }^{50} 51$ Hence, recommended feeding practices follow from observing infant cues for readiness, ${ }^{52} 53$ rather than from assessing oral motor abilities (such as non-nutritive sucking) or from documenting quantity of ingested feeds. ${ }^{53}$ Accordingly, there has been a recent increase in attention to paediatric feeding-swallowing disorders and their relationship to communication development. ${ }^{54-59}$ Research investigations involving swallowing behaviours and feeding are badly needed to guide preventative and clinical treatment initiatives to promote the healthy development of communication from infancy to school entry.

\section{Framework}

Given the breath of our topic, we chose to conduct a scoping review of the literature that will guide knowledge translation, identify gaps in the literature and inform future research investigations from an emerging evidence base. ${ }^{60}$ Our scoping review follows Arskey and O'Malley's framework involving six stages, ${ }^{61}$ including elaborations, such a clearly articulated reason and scope of inquiry as well as a transparent and replicable approach to study selection. ${ }^{62}$ We have applied the Preferred Reporting Items for Systematic Review and Meta-Analysis Protocols reporting checklist ${ }^{6364}$ as a guide for the process of literature appraisal and for reporting results (online supplementary table 1).

\section{Patient and public involvement}

Neither patients nor the public was involved in the development of the current scoping review protocol.

\section{Stage 1: identifying the research questions}

We have developed a single broad research question to inform our search strategy and to derive key domains for our results. We seek to understand the foundational underpinnings of the early development of communication in relation to its inherent physiological and environmental precursors surrounding early swallowing and feeding behaviours. The overarching broad question that our review addresses is:

What early feeding/swallowing factors are associated with communication development from birth to six $(<6 ; 0)$ years of age?

Our line of inquiry includes early child development in both health and congenital or acquired diseases/disorders. The resulting review will facilitate an appreciation of swallowing-feeding factors that relate to the development of communication within an epidemiological framework. Underlying secondary questions relate to swallowing physiology and the feeding environment more specifically. The development of our secondary questions results from an iterative process, whereby reformulations of the questions posed a priori (ie, those identified herein) may result and/or new questions may arise. Our secondary questions currently include:

1. What underlying congenital problems (eg, cerebral palsy, cardiac/respiratory/gastrointestinal anatomical 
Table 4 Basic study characteristics for extraction from each accepted article

\begin{tabular}{|c|c|}
\hline Categories & Characteristics \\
\hline (1) Article details & $\begin{array}{l}\text { Year of publication. } \\
\text { Document type (peer-reviewed journal } \\
\text { article, thesis, government document, } \\
\text { conference proceeding or published } \\
\text { abstract). } \\
\text { Location of study (country). }\end{array}$ \\
\hline (2) Study details & $\begin{array}{l}\text { Design (cohort, case-series, RCT, case } \\
\text { study, case series, qualitative). } \\
\text { Time frame (cross-sectional, } \\
\text { longitudinal). } \\
\text { Setting (hospital, home). } \\
\text { Population (children with typical } \\
\text { communication development, children } \\
\text { with disordered/atypical communication } \\
\text { development). }\end{array}$ \\
\hline $\begin{array}{l}\text { (3) Sample } \\
\text { characteristics }\end{array}$ & $\begin{array}{l}\text { Sample size. } \\
\text { Groups. } \\
\text { Age range. } \\
\text { Sex/gender. } \\
\text { Ethnicity. } \\
\text { Language(s) used. } \\
\text { Aetiology (if applicable). } \\
\text { Comorbidities (if applicable). } \\
\text { Hearing status. }\end{array}$ \\
\hline $\begin{array}{l}\text { (4) Feeding/swallowing } \\
\text { variables }\end{array}$ & $\begin{array}{l}\text { Physiology and behaviours. } \\
\text { Environment. } \\
\text { Modes. }\end{array}$ \\
\hline $\begin{array}{l}\text { (5) Communication } \\
\text { outcomes }\end{array}$ & $\begin{array}{l}\text { Speech (early vocalisations, babbling, } \\
\text { protowords, single word production, } \\
\text { multiword production). } \\
\text { Language (content, form, use). } \\
\text { Gestural behaviours. }\end{array}$ \\
\hline $\begin{array}{l}\text { (5) Main findings of } \\
\text { study }\end{array}$ & $\begin{array}{l}\text { Trends } \\
\text { Effects }\end{array}$ \\
\hline (6) Data analyses & $\begin{array}{l}\text { Type } \\
\text { Rigour }\end{array}$ \\
\hline $\begin{array}{l}\text { (7) Themes/domains } \\
\text { of study }\end{array}$ & $\begin{array}{l}\text { Barriers to communication development } \\
\text { Facilitators to communication development }\end{array}$ \\
\hline (8) Study limitations & $\begin{array}{l}\text { Design } \\
\text { Outcomes } \\
\text { Generalisations }\end{array}$ \\
\hline (9) Pertinence ${ }^{43}$ & $\begin{array}{l}\text { Clinical practice } \\
\text { Policy } \\
\text { Research }\end{array}$ \\
\hline
\end{tabular}

defects, neurological syndromes, prematurity, transient birth-related distress) impact swallowing-feeding integrity and the development of communicative functions?

2. What swallowing-related impairments (eg, poor respiratory function, reflux, weak neonatal latch/sucking behaviours, otitis media) compromise or delay the onset of communicative functions?

3. How do feeding environments alter the onset and course of development of communicative functions?

a. Feeding mode (breast, bottle, combination feeding, enteral feeding).

b. Setting (neonatal intensive care unit stay, hospital stay, interventions such as incubation for phototherapy). c. Interactive constructs (quality and quantity of feeding-related caregiver verbal and/or tactile interactions).

\section{Stage 2: identifying relevant studies}

The search strategy for Medline was developed in three steps by the first (HF) and third authors (ME) with input by the final author (SS) through ongoing consultation with a research librarian. First, we undertook a consultative process, whereby we analysed and reviewed all Medical Subject Heading ( $\mathrm{MeSH}$ ) terms associated with communicative functions and feeding/swallowing behaviours multiple times to maximise specificity while retaining the necessary scope of inquiry. Subsequently, the first and third authors chose MeSH terms for explosion if all subordinate terms were truly relevant to the content area and research questions. Second, review of other relevant systematic or scoping reviews revealed additional possibilities for MeSH terms and context-dependent terms (eg, title, abstract and keywords) in the areas of swallowing-feeding ${ }^{65-68}$ and communication. ${ }^{69-72}$ The two authors (HF and ME) developing the search strategy selected terms for a paediatric population from a published filter ${ }^{73}$ identified by the research librarian, rather than limit the search within Medline. Finally, we developed additional context-dependent terms that we felt were not captured from the previous two steps based on our expert knowledge of the fields of swallowing and communicative sciences. The research librarian then confirmed the integrity of the terms and search strategy (table 1) and provided consultation for additional databases to search.

We selected nine additional databases to search based on adaptations of the Medline search terms (table 2). We did not include PubMed as part of the search, given the possibility of its referencing predatory journals. ${ }^{74}$ We consider our set of selected databases sufficient to capture potentially relevant PubMed citations. We will not apply date or language limits on the searches to the extent possible. That is, we will accommodate languages that we can read (including at least English, French, German, Italian, Persian and Spanish) and seek translations for other languages (if possible) from colleagues and contacts within our scientific communities. All study designs are of interest, including case reports, case series, qualitative, cohort, case-control and quasi-experimental studies, as well as randomised controlled trials.

Further, we identified additional sources most relevant to the content of the scoping review and based on feasibility of search conduct. We will apply the same article inclusion criteria and determine search terms accordingly. Selections include 17 journals, 4 conference proceedings and 6 grey literature sources (table 2). The research librarian assisted with determining grey literature sources. We will search all sources electronically from the first date of their online availability. Finally, we will review the reference lists and citations of selected articles through Google Scholar using a process of forward 
and backward chaining. ${ }^{75}$ Our intent is to complete all searches within 6 months of the publication of the current scoping review protocol.

\section{Stage 3: selecting studies}

We will compile and store all citations using Covidence ${ }^{76}$ and Microsoft Excel. Following elimination of duplicates and citations without abstracts, we will review the remaining abstracts and articles in a two-step process. Two independent reviewers will code the abstracts for potential inclusion or exclusion (table 3 ). Coding criteria are purposefully open-ended for abstract review to permit selection of all those potentially relevant for full article retrieval. Additionally, codes are hierarchical, such that if code 1 is relevant, and the abstract therefore deemed ineligible, the remaining codes are not applicable. Subsequently, the two abstract reviewers will discuss and resolve discrepancies by consensus, requesting input from a third reviewer for consensus deliberations. All abstracts not assigned an exclusion code will be accepted for full review.

The same process will apply to full article review to determine final articles for inclusion. That is, two reviewers, blind to each other's evaluations, will independently assess all full articles, determining eligibility for inclusion according to more stringent and hierarchically coded criteria (table 3). Any articles written in languages outside of those understood by the two reviewers will undergo translation (if possible) for key methodological content to enable coding. Coding criteria for the full article review will be more stringent than for the abstract review, since all pertinent information will be available and reviewers will make a final decision about article selection. Where discrepancies exist, the two reviewers will engage in discussion to achieve consensus regarding inclusion or exclusion. In the event of difficult resolution, a third reviewer will assist in consensus deliberations. That is, all three reviewers will revisit the article in a process of reiterative evaluation to determine final inclusion or exclusion of the article. All codes and final decisions for inclusion or exclusion from both abstract and full article reviews will be entered into the excel datasheet.

Articles without exclusion codes will be accepted for the scoping review. Accepted articles that initially required translation will undergo a second review (if possible) by the same translator and/or a different translator to ensure that the appropriate content is available for data extraction and charting.

\section{Stage 4: charting the data}

Multiple reviewers will be responsible for collecting data, each from a proportion of the articles, followed by independent verification of all data by research personnel. Data collection will involve documenting key characteristics and variables of interest for each article (table 4) and recording them in a database for storage and analysis. All reviewers collecting and charting data will be responsible for ongoing communication about emerging themes or domains of inquiry that could alter and enhance data collection procedures.

\section{Stage 5: collating, summarising and reporting the results}

Data from stage four will be summarised to map the emergence and breadth of literature over time for the primary objective. Subsequently, we will stratify the findings according to healthy vs impaired development. Various forms of data presentation will include tables, line graphs (for chronological information), histograms and/or pie charts. To illustrate, we will tabulate study characteristics according to design, population, setting and pertinence for policy, clinical practice or research. Similarly, we will visually represent information pertaining to swallowing-feeding functions and domains of communication development in charts. Where available, we will describe themes that arise from our review, including barriers and facilitators to the development of communicative functions in young children. We will not include quality or rigour of specific analyses in the scoping review, because the intent is to obtain a sense of the extent of the literature in the area rather than appraise individual articles.

Our scoping review will therefore provide a first overview of trends and breadth for an emerging body of literature. Most important, we will identify gaps in the current knowledge base and provide guidance for the prevention and treatment of potential communication impairments beginning in early infancy.

\section{Stage 6: optional consultation exercise}

We intend to seek input and consultation from caregivers and practitioners once we have compiled and mapped the results of our full scoping review. We hope that such consultation may elucidate gaps that were otherwise not salient to us and subsequently render our knowledge translation endeavours more feasible and accessible to stakeholders.

\section{ETHICS AND DISSEMINATION}

Following completion of all stages of scoping review, findings will be disseminated via local and international conference presentations and submission to a peer-reviewed journal. Knowledge translation activities will then include development of accessible materials for end-users, such as parents, infant caregivers and clinicians from various fields of professional practice, so that they may understand and advocate best practice for communicative development in children. In addition, we will share our findings with policy-making organisations and other stakeholders, such as the Canadian Child and Youth Health Coalition and the Paediatric International Patient Safety and Quality Collaborative.

\section{Author affiliations}

${ }^{1}$ School of Rehabilitation Sciences, Faculty of Health Sciences, University of Ottawa, Ottawa, Ontario, Canada

${ }^{2}$ Institut du savoir - A Knowledge Institute, Montfort Hospital, Ottawa, Ontario, Canada 
${ }^{3}$ In-patient Rehabilitation, Elisabeth-Bruyere Hospital, Ottawa, Ontario, Canada ${ }^{4}$ In-patient Rehabilitation, Children's Hospital of Eastern Ontario, Ottawa, Ontario, Canada

${ }^{5}$ In-patient Medical Units, Hopital Montfort, Ottawa, Ontario, Canada

${ }^{6}$ School of Audiology and Speech Sciences, The University of British Columbia, Vancouver, Ontario, Canada

${ }^{7}$ Faculty of Medicine and Dentistry, University of Alberta, Edmonton, Ontario, Canada

Acknowledgements The authors acknowledge Marie-Cécile Domecq from the Health Sciences Library, Faculty of Health Sciences, University of Ottawa, for her advice and availability for consultation during the development of the current scoping review protocol.

Contributors All coauthors provided meaningful and unique contributions to the development, preparation and/or writing of the current scoping review protocol. HF formulated the topic and conceptualised the questions and theoretical framework. She also guided the research team at all stages of the review development and wrote the bulk of the protocol. DB contributed critical and novel insight for the research questions, theoretical framework and review components relative to communicative functions. SS contributed critical and novel insight for the research questions and for operational definitions relative to swallowing functions. She also provided advice for scoping review methods and proposed analyses. ME provided conceptual input in the development of the search terms relating to communicative functions in collaboration with $\mathrm{HF}$ and in consultation from a research librarian. M-FP contributed to writing the introduction, especially with respect to epidemiology of communication impairments. SM contributed by drafting ideas related to the clinical relevance of the scoping review. All authors reviewed multiple renditions of the manuscript, providing suggestions and editing until all approved the appropriateness for submission.

Funding HF and DB received start-up funding from the Faculty of Health Sciences, University of Ottawa, facilitating the development and future conduct of this scoping review. SS holds start-up funding from the Faculty of Medicine's School of Audiology and Speech Science at the University of British Columbia.

Competing interests Not required.

Patient consent for publication Not required.

Provenance and peer review Not commissioned; externally peer reviewed.

Open access This is an open access article distributed in accordance with the Creative Commons Attribution Non Commercial (CC BY-NC 4.0) license, which permits others to distribute, remix, adapt, build upon this work non-commercially, and license their derivative works on different terms, provided the original work is properly cited, appropriate credit is given, any changes made indicated, and the use is non-commercial. See: http://creativecommons.org/licenses/by-nc/4.0/.

\section{REFERENCES}

1. Hutchon B, Gibbs D, Harniess P, et al. Early intervention programmes for infants at high risk of atypical neurodevelopmental outcome. Dev Med Child Neurol 2019;(11). doi:10.1111/dmcn.14187. [Epub ahead of print: 04 Mar 2019].

2. Irvine B, Luo W, León JA. Congenital anomalies in Canada 2013: a perinatal health surveillance report by the public health agency of Canada's Canadian perinatal surveillance system. Health Promot Chronic Dis Prev Can 2015;35:21-2.

3. Public Health Agency of Canada. Down Syndrome Surveillance in Canada 2005-2013.[Internet]. [cited 2018 July 7]. Available from, 2017. Available: https://www.canada.ca/en/public-health/services/ publications/healthy-living/down-syndrome-surveillance-2005-2013. html

4. Pavri S, Forrest CR. Demographics of orofacial clefts in Canada from 2002 to 2008. Cleft Palate Craniofac J 2013;50:224-30.

5. Christensen D, Van Naarden Braun K, Doernberg NS, et al. Prevalence of cerebral palsy, co-occurring autism spectrum disorders, and motor functioning - Autism and Developmental Disabilities Monitoring Network, USA, 2008. Dev Med Child Neurol 2014;56:59-65.

6. Statistics Canada. Health Fact Sheets Preterm live births in Canada, 2000 to 2013. [Internet], 2016. Available: https://www150.statcan.gc. $\mathrm{ca} / \mathrm{n} 1 / \mathrm{pub} / 82-625-\mathrm{x} / 2016001 / \mathrm{article} / 14675-$ eng.htm [Accessed cited 2017 July 7]

7. Black LI, Vahratian A, Hoffman HJ. Communication disorders and use of intervention services among children aged 3-17 years: United
States, 2012. NCHS data brief, no 205. Hyattsville, MD: National Center for Health Statistics, 2015.

8. Norbury CF, Gooch D, Wray C, et al. The impact of nonverbal ability on prevalence and clinical presentation of language disorder: evidence from a population study. J Child Psychol Psychiatr 2016;57:1247-57.

9. Tomblin JB, Records NL, Buckwalter P, et al. Prevalence of specific language impairment in kindergarten children. $J$ Speech Lang Hear Res 1997;40:1245-60.

10. Beitchman JH, Nair R, Clegg M, et al. Prevalence of speech and language disorders in 5-year-old kindergarten children in the OttawaCarleton region. J Speech Hear Disord 1986;51:98-110.

11. Campbell TF, Dollaghan CA, Rockette HE, et al. Risk factors for speech delay of unknown origin in 3-year-old children. Child Dev 2003;74:346-57.

12. Public Health Agency of Canada. Autism Spectrum Disorder among Children and Youth in Canada 2018. A Report of the National Autism Spectrum Disorder Surveillance System [Internet], 2018. Available: https://www.canada.ca/en/public-health/services/publications/ diseases-conditions/autism-spectrum-disorder-children-youthcanada-2018.html\#a2-7 [Accessed cited 2018 July 7].

13. Morgan L, Wren YE. A systematic review of the literature on early vocalizations and Babbling patterns in young children. Commun Disord Q 2018;40:3-14.

14. AlHammadi FS. Prediction of child language development: a review of literature in early childhood communication disorders. Lingua 2017;199:27-35.

15. Etchell A, Adhikari A, Weinberg LS, et al. A systematic literature review of sex differences in childhood language and brain development. Neuropsychologia 2018;114:19-31.

16. Fisher EL. A systematic review and meta-analysis of predictors of expressive-language outcomes among late talkers. J Speech Lang Hear Res 2017;60:2935-48.

17. Matthews D, Biney $H$, Abbot-Smith $\mathrm{K}$. Individual differences in Children's pragmatic ability: A review of associations with formal language, social cognition, and executive functions. Language Learning and Development 2018;14:186-223.

18. Carvalho MES, de Miranda Justo JMR, Gratier M, et al. The impact of maternal voice on the fetus: a systematic review. Curr Womens Health Rev 2019;15:196-206.

19. Ferrari GA, Nicolini Y, Demuru E, et al. Ultrasonographic investigation of human fetus responses to maternal communicative and noncommunicative stimuli. Front Psychol 2016;7:354.

20. Yoo H, Bowman DA, Oller DK. The origin of protoconversation: an examination of caregiver responses to Cry and speech-like vocalizations. Front Psychol 2018;9.

21. Stark RE, Rose SN, Benson PJ. Classification of infant vocalization. Int J Lang Commun Disord 1978;13:41-7.

22. Emde Ret al. The moral self of infancy: affective core and procedural knowledge. Developmental Review 1991;11:251-70.

23. Sroufe LA, Coffino B, Carlson EA. Conceptualizing the role of early experience: lessons from the Minnesota longitudinal study. Dev Rev 2010;30:36-51.

24. Easterbrooks MA, Bureau J-F, Lyons-Ruth K. Developmental correlates and predictors of emotional availability in mother-child interaction: a longitudinal study from infancy to middle childhood. Dev Psychopathol 2012;24:65-78.

25. Bigelow AE, Power M, MacLean K, et al. Mother-Infant skin-toskin contact and mother-child interaction 9 years later. Soc. Dev. 2018;27:937-51

26. Page M, Wilhelm MS, Gamble WC, et al. A comparison of maternal sensitivity and verbal stimulation as unique predictors of infant social-emotional and cognitive development. Infant Behav Dev 2010;33:101-10.

27. Bowers $\mathrm{K}$, Khoury J, Sucharew $\mathrm{H}$, et al. Early infant attention as a predictor of social and communicative behavior in childhood. Int $J$ Behav Dev 2019:43:204-11.

28. Loy M, Masur EF, Olson J. Developmental changes in infants' and mothers' pathways to achieving joint attention episodes. Infant Behav Dev 2018;50:264-73.

29. Chang L, de Barbaro K, Deák G. Contingencies between infants' gaze, vocal, and manual actions and mothers' Object-Naming: longitudinal changes from 4 to 9 months. Dev Neuropsychol 2016;41:342-61.

30. Stevenson RD, Allaire $\mathrm{JH}$. The development of normal feeding and swallowing. Pediatr Clin North Am 1991;38:1439-53.

31. Owens RE. Language development: an introduction. $9^{\text {th }}$ ed. Pearson/ Allyn and Bacon, 2016: 1-126.

32. Davies WH, Satter E, Berlin KS, et al. Reconceptualizing feeding and feeding disorders in interpersonal context: the case for a relational disorder. J Fam Psychol 2006;20:409-17. 
33. Koopmans-van Beinum F VderS. Early stages in infant speech development. Proceedings of the Institute of Phonetic Sciences 1979;5:30-43.

34. Koopmans-van Beinum F, Van der Stelt JM. Early Stages in the Development of Speech Movements. In: Lndblom B, Zetterström $\mathrm{R}$, eds. Precursors of Early Speech: Proceedings of an international symposium held at the Wenner-Gren Center, Stockholm, September 10-22, 1984. Basingstoke: Macmillan, 1986: 37-50.

35. Oller DK, Wieman LA, Doyle WJ, et al. Infant babbling and speech. $J$ Child Lang 1976;3:1-11.

36. Oller DK. The emergence of the speech capacity. Mahwah, NJ: Lawrence Erlbaum Assoicates, Publishers, 2000.

37. Bernhardt M, Stemberger J, Charest M. Intervention for speech production in children and adolescents: models of speech production and therapy approaches. Introduction to the issue. Can $J$ Speech Lang Pathol Audiol 2010;34:157-67.

38. Munakata Y, McClelland JL. Connectionist models of development Dev Sci 2003;6:413-29.

39. Nixon GM, Charbonneau I, Kermack AS, et al. Respiratoryswallowing interactions during sleep in premature infants at term. Respir Physiol Neurobiol 2008;160:76-82.

40. Paydarfar D, Gilbert RJ, Poppel CS, et al. Respiratory phase resetting and airflow changes induced by swallowing in humans. J Physiol 1995;483:273-88.

41. Selley WG, Ellis RE, Flack FC, et al. Coordination of sucking, swallowing and breathing in the newborn: its relationship to infant feeding and normal development. Int $J$ Lang Commun Disord 1990;25:311-27.

42. Duvallet C, Larson K, Snapper S, et al. Aerodigestive sampling reveals altered microbial exchange between lung, oropharyngeal, and gastric microbiomes in children with impaired swallow function. PLoS One 2019;14:e0216453.

43. Rawool VW. Prevalence of auditory problems in children with feeding and swallowing disorders. J Speech Lang Hear Res 2017;60:1436-47.

44. Yüksel F, Doğan M, Karataș D, et al. Gastroesophageal reflux disease in children with chronic otitis media with effusion. J Craniofac Surg 2013;24:380-3.

45. Cai T, McPherson B. Hearing loss in children with otitis media with effusion: a systematic review. Int J Audiol 2017;56:65-76.

46. Rommel N, Veereman G. GERD and Dysphagia. In: Vandenplas Y, ed. Gastroesophageal reflux in children. Springer international publishing, 2017.

47. World Health Organization. International Classification of Diseases for Mortality and Morbidity Statistics. 11th Revision, Reference Guide. [Internet], 2018. Available: https://icd.who.int/browse11//-m/ en\#http\%3a\%2f\%2fid.who.int\%2ficd\%2fentity\%2f1412387537 [Accessed cited 23 Nov 2018].

48. Delaney AL, Arvedson JC. Development of swallowing and feeding: prenatal through first year of life. Dev Disabil Res Rev 2008;14:105-17.

49. Pinker S. Out of the minds of babes. Science 1999;283:40-1.

50. Nugent JK, Bartlett JD, Von Ende A, et al. The effects of the newborn behavioral observations (NBO) system on sensitivity in Mother-Infant interactions. Infants \& Young Children 2017;30:257-68.

51. Barlow J, Herath NI, Bartram Torrance C, et al. The neonatal behavioral assessment scale (NBAS) and newborn behavioral observations (NBO) system for supporting caregivers and improving outcomes in caregivers and their infants. Cochrane Database Syst Rev 2018;3.

52. Shaker CS. Cue-based feeding in the NICU: using the infant's communication as a guide. Neonatal Netw 2013;32:404-8.

53. Whetten $\mathrm{CH}$. Cue-Based feeding in the NICU. Nurs Womens Health 2016;20:507-10.

54. Adams-Chapman I, Bann CM, Vaucher YE, et al. Association between feeding difficulties and language delay in preterm infants using Bayley scales of infant Development-Third edition. $J$ Pediatr 2013;163:680-5.
55. Barnevik Olsson M, Carlsson LH, Westerlund J, et al. Autism before diagnosis: crying, feeding and sleeping problems in the first two years of life. Acta Paediatr 2013;102:635-9.

56. Girard L-C, Doyle O, Tremblay RE. Breastfeeding, cognitive and noncognitive development in early childhood: a population study. Pediatrics 2017;139:e20161848

57. Malas K, Trudeau N, Chagnon M, et al. Feeding-swallowing difficulties in children later diagnosed with language impairment. Dev Med Child Neurol 2015;57:872-9.

58. Malas K, Trudeau N, Giroux M-C, et al. Prior history of FeedingSwallowing difficulties in children with language impairment. Am J Speech Lang Pathol 2017;26:138-45.

59. Palladino RRR, Cunha MC, Souza LAdeP, de Paula Souza LA. [Language and eating problems in children: co-occurrences or coincidences?]. Pro Fono 2007;19:205-14.

60. Joanne Briggs Institute. Reviewer's Manual 2015: Methodology for JBI Scoping Reviews. 2015: Joanne Briggs Institute, Australia. Available: http://joannabriggs.org/assets/docs/sumari/ReviewersManual_Methodology-for-JBI-Scoping-Reviews_2015_v2.pdf [Accessed 7 Jul 2018].

61. Arksey H, O'Malley L. Scoping studies: towards a methodological framework. Int J Soc Res Methodol 2005;8:19-32.

62. Levac D, Colquhoun H, O'Brien KK. Scoping studies: advancing the methodology. Implementation Sci 2010;5.

63. Moher D, Shamseer L, Clarke M, et al. Preferred reporting items for systematic review and meta-analysis protocols (PRISMA-P) 2015 statement. Syst Rev 2015;4.

64. Shamseer L, Moher D, Clarke M, et al. Preferred reporting items for systematic review and meta-analysis protocols (PRISMA-P) 2015: elaboration and explanation. BMJ 2015;349.

65. Bessell A, Hooper L, Shaw WC, et al. Feeding interventions for growth and development in infants with cleft lip, cleft palate or cleft lip and palate. Cochrane Database Syst Rev 2011;94.

66. Morgan AT, Dodrill P, Ward EC, et al. Interventions for oropharyngeal dysphagia in children with neurological impairment. Cochrane Database Syst Rev 2012;11.

67. Psaila K, Foster JP, Richards R, et al. Non-Nutritive sucking for gastro-oesophageal reflux disease in preterm and low birth weight infants. Cochrane Database Syst Rev 2014;10:1-13.

68. Tighe M, Afzal NA, Bevan A, et al. Pharmacological treatment of children with gastro-oesophageal reflux. Cochrane Database Syst Rev 2014;11.

69. Brignell A, Song $\mathrm{H}$, Zhu J, et al. Communication intervention for autism spectrum disorders in minimally verbal children. Cochrane Database Syst Rev 2016;63.

70. Law J, Dennis JA, Charlton JJV, et al. Speech and language therapy interventions for children with primary speech and/or language disorders. Cochrane Database Syst Rev 2017;47.

71. Lee AS-Y, Gibbon FE, Cochrane Developmental, Psychosocial and Learning Problems Group. Non-speech oral motor treatment for children with developmental speech sound disorders. Cochrane Database Syst Rev 2015;46.

72. Morgan AT, Vogel AP. A cochrane review of treatment for childhood apraxia of speech. Eur J Phys Rehabil Med 2009;45:103-10.

73. Desmeules R. Filter to Retrieve Pediatric Articles in the OVID Medline Database. John W Scott Health Sciences Library, University of Alberta. [Internet]. Available: Filter-20to-20Retrieve-20Pediatrics20Articles-20in-20OVID-20EMBASE in era.library.ualberta.ca [Accessed cited 2018 Nov 23].

74. Manca A, Moher D, Cugusi L, et al. How predatory journals leak into PubMed. Can Med Assoc J 2018;190:E1042-E1045.

75. Booth A. Unpacking your literature search toolbox: on search styles and tactics. Health Info Libr J 2008;25:313-7.

76. Covidence systematic review software, Veritas Health Innovation, Melbourne, Australia. Available: www.covidence.org [Accessed Aug 2018]. 